\title{
Líneas y modelos de investigación en el actual contexto histórico de El Salvador y Centroamérica ${ }^{\prime}$
}

\author{
Héctor Samour \\ Viceministro de Educación
}

\section{Señoras y señores:}

Muchas gracias a la Universidad Centroamericana "José Simeón Cañas", por compartir con el Ministerio de Educación este momento académico trascendente para la vida interna de la UCA y a su Misión y Visión académica de compromiso que ha mostrado en toda su existencia para el rumbo de la educación superior de El Salvador que busca nuevos caminos de transformación y para el desarrollo social y económico del país.

El momento académico universitario y el evento de hoy tienen un significado extraordinario que quisiera precisar con mayor detenimiento. En primer lugar, con esta acción la UCA muestra que ha asumido el reto actual que enfrentan las instituciones de educación superior en todo el mundo, como es introducirse al mundo de la producción del conocimiento e incorporarse a las exigencias que en la actualidad el desarrollo de la ciencia, la tecnología y la innovación $-y$ en esto debemos reconocer la presencia de la globalizaciónimponen a los países en el mundo entero.

En segundo lugar, al retomar el desafío de la producción del conocimiento, la UCA abre una vía para superar en las instituciones de educación superior de nuestro país, la tradicional acción de transferir y repetir conocimientos producidos en otros países, en otras realidades, de acuerdo a modos de conocer que respondían a necesidades propias de sociedades situadas en sus fases de desarrollo originales.

La UCA, y aquí presto una figura ajena, se ha hecho una pregunta crucial ante la difícil situación del país y del mundo, del Sur y del Norte, del desarrollo y del subdesarrollo y que se plantea en cómo hacer una nueva alianza, un nuevo pacto social con la ciencia frente a las necesidades e intereses de país y de los sectores marginados por la desigualdad y la falta de oportunidades. Es decir, la UCA da un seguimiento a la visión de ese gran pensador que fue Ignacio 
Ellacuría, quien planteó que era necesario producir, en todos los ámbitos de las ciencias, conocimientos innovadores, pertinentes y comprometidos con la democracia y con la conformación de procesos económicos y tecnológicos para superar la injusticia social y la exclusión social.

En tercer lugar, la UCA es la primera, y creo la única, universidad en El Salvador que ha dedicado un fondo específico -de su presupuesto propio y no de la cooperación internacional o como venta de servicios de conocimientos- para desarrollar el área de investigaciones. Es una iniciativa ejemplar que subirá los niveles de competencia positiva entre las instituciones de educación superior y ayudará a mejorar la calidad educativa y su gestión académica.

Al dar este paso, la UCA misma está frente a otro reto importante; prepararse para cambiar las formas de transferencia de conocimientos con sus estudiantes y con los solicitantes de sus servicios en el exterior, como las empresas públicas y privadas. Esto conlleva a profundizar los cambios en la formación y educación continua de sus docentes, y a mantener los recursos educativos que conllevan estos cambios de acuerdo a las nuevas exigencias. Significa, en el mediano y largo plazo, un paquete académico de primer orden, un cambio educativo en toda la extensión de la palabra, un nuevo "modo de producir conocimiento", para usar la terminología que ha impuesto el profesor Michael Gibbons desde el año 1994 (Gibbons et al., 1994).

Aquí debo recordar un poco la experiencia que la UCA realizó en los últimos treinta años del siglo pasado en el campo de la investigación, que fue muy destacada y de grandes alcances para el país. Es posible decir que la UCA, desde los años 70 había dejado atrás, y por mucho, el "Modo 1" de producción de conocimientos que aún impera en muchas de nuestras universidades, y en las de otros países, un modo más ligado a la Europa de la época de la consolidación del capitalismo, la emergencia del pensamiento humanista ilustrado y el debilitamiento de las monarquías absolutistas.

Los académicos de ese período también se esforzaron por generar conocimiento para el progreso social, controlar y predecir desastres naturales, incluso comprender los procesos sociales y aportar para superar injusticias, recordemos a Marx, por ejemplo. Sin embargo, lo hicieron desde un tipo de ciencia disciplinar que solo aceptaba sus propias metodologías y formas de validación, sin relacionarse e integrarse con las otras ciencias. Eran procesos que recuerdan el trabajo solitario de los artesanos de la ciencia, casi con la organización de producción y organización del 
taller artesanal. Nos recuerdan a los docentes actuales que comparten el tiempo de la docencia con escasas horas en sus casas para avanzar en la investigación individualizada y disciplinar, con la que satisfacen sus inquietudes de producir conocimiento nuevo.

La UCA, en el período mencionado, ya había organizado investigaciones altamente complejas para desarrollar el país y mejorar las condiciones de vida de los sectores más débiles de la sociedad. Recuerdo una primera gran investigación sobre las necesidades de hacer avanzar la producción y distribución de la energía en el sector rural del país para que pudiese ampliarse su uso productivo, público y domiciliar. Una investigación hecha con el Banco Mundial. Equipos de economistas, ingenieros, administradores de empresas, sociólogos, informáticos que trabajaron en tareas multidisciplinarias y transdisciplinarias.

Con esta misma visión, la UCA también trabajó en una investigación sobre el desarrollo del sector agrario del país, investigando aspectos básicos de las formas de producir, del comercio nacional e internacional de sus productos, aspectos económicos, sociales y políticos con la idea de aportar información y usos de la misma para crear políticas de cambio en el agro. Hizo estudios sobre aspectos electorales y de producción de energía en ríos pequeños para producir y hacer progresar a comunidades pequeñas. Lo fundamental de esta producción de conocimiento era la proyección social de la universidad hacia las comunidades más pobres del país, para sensibilizarlas y formarlas con un pensamiento y acción independiente hacia una nueva participación social y política, que le permitiera mejorar sus vidas. Se planteaban los resultados y proyectos a los otros sectores de la población, a empresarios, al Estado, entre otros.

Estas iniciativas están más cercanas al "Modo 2" de Gibbons, ligado al contexto de aplicación como su principal atributo ${ }^{2}$. El conocimiento era especializado, de calidad y útil para la sociedad, el gobierno y la gente. El conocimiento se producía bajo una negociación continua y la UCA se mantenía siempre preguntando y exigiendo que se incluyeran los intereses de los sectores menos privilegiados. "¿Desarrollo para qué?, ¿Desarrollo para quién?" se decía en aquella época y se sigue diciendo ahora. Aquí, el conocimiento ya no se realizaba solo desde el tenor de una disciplina, sino con el concurso sinérgico de las diversas formas del saber. Había un saber transdisciplinar con sus propias estructuras teóricas individuales, sus peculiares métodos de investigación y sus prácticas investigativas. Había mucha discusión 
y propuestas explicativas con sus respectivas soluciones.

La forma de producir conocimiento en el "Modo 2" ha dejado a las universidades como lugares exclusivos de producción de conocimientos y se ha expandido hacia los centros de investigación, empresas privadas, incluso en Ministerios públicos, Ong's, compañías asesoras y otros. La Fundación Salvadoreña para el Desarrollo Económico y Social (FUSADES), La Fundación Nacional para el Desarrollo (FUNDE), La Fundación Guillermo Manuel Ungo (FUNDAUNGO) y otras entidades, son un ejemplo en el país. Tienen una forma variada de intercomunicarse entre ellos desde la electrónica, la social y hasta la informal. Pero lo fundamental es que la investigación disciplinar ha dejado de ser su forma de actuar para dar paso a reconfiguraciones donde la flexibilidad y el tiempo marcan los ritmos de entrega de sus productos, aunque la mayoría de las veces solo hay descripciones o síntesis de conocimientos previos, pero no se presentan en realidad nuevos conocimientos.

Pero ahora se está perfilando un nuevo modo de conocimiento, el "Modo 3", que está bajo un nuevo tipo de relaciones epistemopolíticas para una nueva fase de realidad en formación y que está cambiando ideas, conceptos, formas de pensar las cosas, el mundo y que puede abrir una ruta nueva de producir conocimiento (Valdeleón \& Carreño Manosalva, 2010). Se está construyendo en el marco de la sociedad en riesgo al lado de las grandes crisis planetarias que inciden en cada uno de nuestros países y regiones. Se hace presente, por ejemplo, en el problema universal de la crisis ecológica del globo, producto de la degradación ambiental provocada por el capitalismo industrial. Otro tema global es la imposibilidad, hasta ahora, de diseñar y concretar una globalización alternativa, que ha llevado a algunos a afirmar que "solo hay un capitalismo sin final", que lleva implícita una denuncia al consumismo y al avance de la tecnología en la extracción, producción, distribución, consumo y deshechos de los productos. A su vez, es también una crítica a quienes producen conocimiento. El calentamiento global como las crisis financieras mundiales, los cambios en las nuevas relaciones productivas internacionales, la revolución científica mundial y la búsqueda de nuevas fuentes de energía, son el contexto de la nueva forma de investigación y de producción de conocimiento.

La idea de la UCA de mantener siempre la presencia de los agentes sociales excluidos es una de las características más importantes de este "Modo 3", que dice que desde la producción del conocimiento también se puede denunciar y, 
quizás más importante, construir alternativas para el capitalismo depredador y para que la creatividad humana sea puesta al servicio de una convivencia antagónica al lucro que promociona al máximo el consumo irresponsable y degradante para la dignidad humana.

Sostener la presencia de los actores sociales, distintos a los del gobierno y de la empresa privada, en el proceso de producción del conocimiento genera formas más democráticas de acceso al conocimiento y dinamiza exponencialmente los procesos de innovación $y$, sobre todo, da origen a nuevas relaciones de producción de conocimiento.

El "Modo 1" era disciplinar, el "Modo 2" era transdisciplinar, ¿qué es ahora el "Modo 3"? Se dice que es "transcultural": el estudio de las problemáticas de las comunidades, desde todas las perspectivas científicas, de materiales que la ciencia, sobre todo la positivista, ha desechado siempre. Me refiero a otras formas de conocimiento de las culturas del mundo que muestran su potencia cognoscitiva en la actualidad y que lentamente están siendo integradas a la "ciencia occidental". Recuerdo hace muchos años cuando en México, Johang Galtung, planteaba que la epistemología debía incorporar las cosmogonías, por ejemplo el "yin yang", en sus formas explicativas, de comprensión y explicación del mundo. Lejos de la episteme disciplinar o transdisciplinar, el saber trata ahora de ser una fusión de conocimientos de diversas tradiciones culturales, rompiendo con el eurocentrismo de la ciencia dominante. Se dice ahora "que la innovación social comunitaria, por ejemplo, frente a una demanda específica logra hacer una síntesis entre el conocimiento tradicional de las comunidades y las diferentes disciplinas científicas para encontrar una solución eficiente y escalable a un determinado problema social" (Valdeleón \& Carreño Manosalva, 2010, p. 79).

Así como lo pensaba Ellacuría, la producción de conocimiento tiene una dimensión ética, política, religiosa, económica y cultural que nos permite ver una realidad en su totalidad, en su complejidad y en su concreción histórica, que va más allá de lo que la ciencia ha logrado conocer.

La investigación actual debe tener una capacidad para comprender lo local, lo concreto, su interacción sin jerarquías con la globalización, para articular propuestas de desarrollo territorial y trabajar las teorías en la práctica, sin absolutizarlas.

El abordaje de la ciencia no es completo si no se toma en cuenta la complejidad y la dinámica de las construcciones sociales, políticas y económicas que constituyen el 
proceso histórico local, nacional y global. Es el proceso social el que impulsa el desarrollo de la ciencia, la tecnología y la innovación y, por lo tanto, sus productos deben estar en función de los movimientos sociales que resisten y protagonizan procesos emancipadores en los diversos ámbitos de la vida social.

\section{Referencias}

- Gibbons, M., et al. (1994). The New Production of Knowledge. London: SAGE

- Gibbons, M. (2000). Mode 2. Society and the Emergence of ContextSensitive Science. Science and Public Policy, Vol. 27. Beech Tree Publishing. pp. 159-163.

- Valdeleón, W. A. \& Carreño Manosalva, C. (2010). Modo 3 de la producción del conocimiento: Implicaciones para la universidad de hoy. Revista de la Universidad Lassalle, No. 61. Bogotá, Colombia, pp. 67-86.

Notas

1 Discurso pronunciado en la apertura del Seminario Fondo de Investigación UCA 2012-2013, Auditorio Segundo Montes, 3 de octubre de 2013, Universidad Centroamericana "José Simeón Cañas".

2 A mediados de los noventa algunos autores británicos encabezados por Michael Gibbons propusieron la aparición de un nuevo modo de producción de conocimiento al que llamaron Modo 2 (M2). De acuerdo a su enunciado, este modo emergente es transdisciplinario, no amarrado a jerarquías organizacionales, basado en el contacto social entre los involucrados y es eminentemente reflexivo. Este tipo de investigación es realizado en el "contexto de la aplicación”, es decir que su principal fundamento es la existencia de la necesidad social desde la más temprana etapa de la investigación en la búsqueda de soluciones. El modo de producción de conocimiento previo y que se opone a este es el Modo 1 (M1), el cual descansa en el conocimiento académico producido en el marco de contextos disciplinarios autónomos; en este tipo de investigación no existe necesariamente una conexión directa entre la investigación misma y la aplicación social, por lo tanto, los límites entre las universidades y la sociedad son claras, por ello el ámbito académico de la investigación era autónomo al escoger sus tópicos, problemas y líneas de investigación (Gibbons et al, 1994). 